\title{
WHEN CLINICAL JUDGEMENT OF A HEART MURMUR IS WRONG: A CASE REPORT
}

\author{
LĂCRĂMIOARA-ELIZA POP ${ }^{1}$, ANGELA BUTNARIU ${ }^{2}$ \\ ${ }^{1}$ Emergency Institute for Cardiovascular Diseases and Transplantation, Târgu Mureş, \\ ${ }^{2}$ Iuliu Hatieganu University of Medicine and Pharmacy, Cluj-Napoca
}

Keywords: congenital heart defect, innocent murmur, transthoracic echocardiography
Abstract: Background: Infants with congenital heart defects (CHD) are at risk of death if not recognized and treated on time, desirable during intrauterine life or soon after birth. Case: A 6-week old infant presented for the evaluation of an innocent murmur, discovered immediately after birth and recorded by the neonatologist, family physician and pediatrician at follow-up visit. At physical examination, a grade 2/6 systolic murmur and tachycardia were observed. The electrocardiography revealed sinus tachycardia. Transthoracic echocardiography recorded multiple heart defects (three ventricular septal defects, an atrial septal defect, valvular regurgitations, impaired right heart function) and a vascular defect. Treatment consisted of surgical repair. Conclusions: The rarity of the case results from the atypical presentation of the cardiovascular defects only through an innocent heart murmur, therefore criteria requested by guidelines for evaluation through transthoracic echocardiography for the suspicion of a CHD, were not encountered and the diagnosis was delayed.

\section{INTRODUCTION}

Infants with congenital heart defects (CHD) are at risk of death if not recognized and treated on time.(1,2) There are published guidelines that could help the clinician to diagnose a child with congenital heart disease, but sometimes diagnostic process could be challenging, as in the reported case.

Many patients are first addressed in the primary care setting, so primary care providers play a key role in diagnosis, management and referral of children with CHD. Clinical judgement is the main tool on which they rely in the assessment of a heart murmur.

The best diagnostic tool for a CHD is transthoracic echocardiography (TTE) but it is not cost-efficient to screen with TEE all children that present a heart murmur.(3) We reviewed the literature in searching for some useful tools that could rise the suspicion accuracy of CHD in children with heart murmur.

Presence of symptoms like effort intolerance, breathing difficulty, palpitation, cyanosis, chest pain, symptoms of heart failure along with clinical findings of loud systolic murmur (more than $3 / 6$ in intensity), diastolic murmur, abnormal heart sounds, abnormal blood pressure all point towards the presence of heart disease.(4) Rarely, the malformation is silent or reduced to a heart murmur.

\section{AIM}

Through this case report we would like to emphasize the limitations of the clinical evaluation of a heart murmur.

\section{CASE PRESENTATION}

The case of a boy aged 6 weeks who was admitted in the Pediatrics Department for a detailed evaluation of a heart murmur is reported. There was no prenatal suspicion of cardiac malformation on fetal ultrasonography. He was the first child in the family, from a normal pregnancy, delivered on term through caesarian section due to pelvic presentation, with normal weight and length and an Apgar score of 10.

At physical examination in the nursery he presented a heart murmur. The murmur was considered "benign", therefore the patient was discharged with no further recommendations. The patient was evaluated by the family physician who recorded the murmur in the patient chart but considered that this murmur has normal characteristics. At one month of life, the parents request a pediatric consultation wanting to know more about the murmur. The pediatrician evaluated the baby and recorded in his chart an innocent heart murmur and a heart rate close to the upper limit for the age. She did not consider that other diagnostic studies are necessary and recommended follow-up. At the age of 6 weeks, the concerned parents brought the patient to the Pediatrics Department for re-evaluation.

At physical examination, a grade $2 / 6$ systolic murmur, best heard on the entire left sternal border and tachycardia were observed. Due to the presence of tachycardia, further investigations were requested in concern for a CHD. The electrocardiography (ECG) revealed sinus tachycardia with a heart rhythm of 180 beats/min (above $90^{\text {th }}$ percentile for age), QRS axis at 100 grades and a high $\mathrm{R}$ in V1 lead (normal features for the age).

The chest x-ray (CXR) revealed cardiomegaly, pulmonary artery dilatation and increased pulmonary vascularization. The TEE recorded: hyperkinetic heart with a large subaortic ventricular septal defect measuring $9 \mathrm{~mm}$ with left to right shunt, partially covered by the septal cusp of tricuspid valve which formed a subaortic membrane without obstructing the blood flow, aorta overriding the ventricular septum, mild aortic insufficiency, two apical muscular

${ }^{1}$ Corresponding author: Lăcrămioara-Eliza Pop, Str. Jupiter, Nr. 1, Cluj-Napoca, România,E-mail: lacri_lizy@yahoo.com, Phone: +40744181011 Article received on 28.06.2020 and accepted for publication on 10.08.2020 


\section{CLINICAL ASPECTS}

ventricular defects with left to right shunt, an atrial septal defect measuring $4 \mathrm{~mm}$ with left to right shunt, left heart volume overload, mild mitral insufficiency, enlarged right ventricle with tricuspid regurgitation, a dilated pulmonary artery, severe pulmonary hypertension, increased flow through pulmonary veins and anomalous origin of left common carotid artery from brachiocephalic trunk (figure no. 1).

Figure no. 1. The multitude of heart defects which manifested only as a grade 2 systolic heart murmur - green boxes represent location of defects - adaptation after Children's Hospital of Philadelphia (RA - right atrium, RV - right ventricle, LA - left atrium, LV - left ventricle, AO aorta, PA - pulmonary artery)

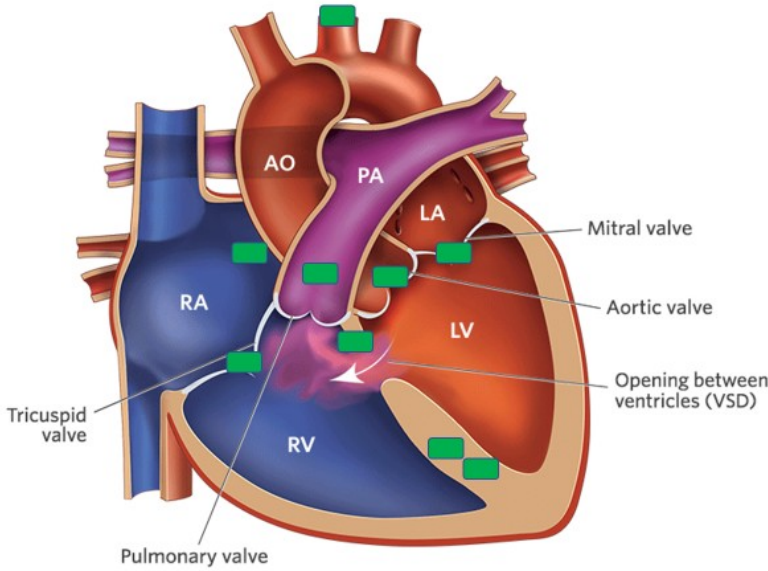

The final diagnosis consisted of multiple cardiac defects and a vascular malformation. Treatment was started with angiotensin converting enzyme inhibitors (enalapril) and diuretics (furosemide, spironolactone) in association with high caloric intake, vitamins, anemia prophylaxis, low sodium diet and shortly after, surgical correction followed. Consent for presentation of the case was obtained from the parents.

\section{DISCUSSIONS}

Cardiac murmurs in children are a frequent finding on physical examinations and most of them are innocent. It is well known that the discovery of a murmur may provoke significant parental anxiety.(5) Studies have reported that up to $50 \%$ of children may have cardiac murmurs, but less than $1 \%$ present an underlying structural heart disease.(6) A parent who finds out that his child has a heart murmur thinks that the child has a serious condition, despite the reassuring epidemiological data offered by the doctor. That was the situation in the presented case. The parents were so anxious about the heart murmur of their child that they sought medical opinion through several consultations (the family physician, the pediatrician - two times).

It is important to discriminate between innocent murmurs and those related to heart disease. Clinical differential implies considerable practice and extensive experience in auscultation. In order to help in the process of discrimination, Computer Aided Detection (CAD) systems were developed.(7) The method can detect very efficiently (average accuracy of $92.6 \%)(7)$ the heart murmur and classify it as innocent or associated to a cardiovascular disease.

The use of electronic or digital stethoscopes with spectral display capabilities has also been proposed as a method to improve the characterization of heart murmurs by cardiac auscultation.(8) One of these newer techniques might have helped in our case.

There are several types of innocent murmur that need to be differentiate from those secondary to a cardiac defect: Still's murmur, innocent pulmonary blood flow murmur, innocent pulmonary branch murmur of the infant, supraclavicular bruit, venous hum, mammary souffle, and cardiorespiratory murmur.(9)

A model proposed in the literature, "Seven S Murmurs" (10) was designed as a useful tool in recognizing an innocent murmur and its semiotic aspects: systolic (occurs during and is limited to systole), sensitive (changes with child's position or with respiration), short duration (not holosystolic), single (no clicks or gallops associated), small (murmur limited to a small area and not irradiating), soft (low amplitude), sweet (not harsh).(11) In our case, the patient presented all the characteristics listed above therefore no supplementary examinations were ordered.

Tachycardia was the red flag in our case and its presence suggested the need of a TEE. The most common causes of neonatal tachycardia are sinus tachycardia and supraventricular tachycardia (SVT).(12) Both are narrowed QRS complexes tachycardia. Sinus tachycardia usually presents with a rate of 160 to 200 beats per minute and SVT usually presents with a rate of 220 to 300 beats per minute. Causes of sinus tachycardia, other than a cardiac defect, include fever, pain, intense crying, infection, anemia, hyperthyroidism or drugs. It is important to distinguish supraventricular tachycardia from sinus tachycardia because SVT is always a problem and has pathological substrates: accessory pathway, AV nodal re-entrant circuits.(13) TTE is the most important, most accessible noninvasive diagnostic procedure for the initial confirmation of suspected congenital cardiovascular malformation. Guidelines recommend TEE for the evaluation of patients with early, late, or holosystolic murmurs and patients with grade 3 or louder mid-systolic murmurs.

Patients with grade 1 or 2 mid-systolic murmurs should be evaluated only when other symptoms or signs of cardiovascular disease are present, including those from ECG or chest x-ray.(14) In the presented case, at the first visit to the pediatrician, there were no reasons for recommending a TEE: the murmur had innocent characteristics and the heart rate was in the normal range. Only at the second visit to the pediatrician, according to guidelines, a TEE was requested and revealed the heart and vascular malformation.

Controversy exists about the usefulness of the CXR and ECG in diagnosing CHD. Adding a CXR and ECG to the pediatrician's evaluation increases costs with little gain in accuracy. Whether the optimal strategy involves referring to a cardiologist or obtaining a TTE for all patients with murmurs depends on how much a medical society would allocate to diagnose pathologic murmurs.(15)

\section{CONCLUSIONS}

Through this case report we would like to emphasize the limitations of the clinical evaluation of a heart murmur based on a case in which the diagnosis was delayed because the patient did not encounter the precise criteria requested by guidelines in order to be evaluated through TTE for the suspicion of a CHD.

The poor clinical picture (heart murmur and tachycardia) of the multiple heart defects of the patient also contributed to a delayed diagnosis.

\section{Acknowledgments:}

The authors wish to thank Daniela Iacob, Simona Oprita and Manuel Chira for their help in the diagnosis and management of the patient

1. Mahle WT, Martin GR, Beekman RH 3rd, Morrow WR. 
Section on Cardiology and Cardiac Surgery Executive Committee. Endorsement of Health and Human Services recommendation for pulse oximetry screening for critical congenital heart disease. Pediatrics. 2012;129(1):190-192.

2. Eckersley L, Sadler L, Parry E, Finucane K, Gentles TL. Timing of diagnosis affects mortality in critical congenital heart disease. Arch Dis Child. 2016;101(6):516-520.

3. Frank JE, Jacobe KM. Evaluation and management of heart murmurs in children. Am Fam Physician. 2011;84(7):793800.

4. Mirzarahimi M, Saadati H, Doustkami H, Alipoor R, Isazadehfar K, Enteshari A. Heart murmur in neonates: how often is it caused by congenital heart disease?. Iran J Pediatr. 2011;21(1):103 - 106

5. Bårdsen T, Sørbye MH, Trønnes H, Greve G, Berg A Parental anxiety related to referral of childhood heart murmur; an observational/interventional study. BMC Pediatr. 2015;15:193. Published 2015 Nov 21.

6. Newburger JW, Rosenthal A, Williams RG, Fellows K, Miettinen OS. Noninvasive tests in the initial evaluation of heart murmurs in children. N Engl J Med. 1983;308:61-64.

7. Ahmad MS, Mir J, Ullah MO, Shahid MLUR, Syed MA. An efficient heart murmur recognition and cardiovascular disorders classification system. Australas Phys Eng Sci Med. 2019;42(3):733-743.

8. Dahl LB, Hasvold P, Arild E, Hasvold T. Heart murmurs recorded by a sensor based electronic stethoscope and emailed for remote assessment. Arch Dis Child. 2002;87(4):297-301.

9. Danford DA. Heart murmur in a child. Case study and commentary. J Clin Outcomes Manag. 2002;9:146-158.

10. Bronzetti G, Corzani A. The Seven "S" Murmurs: an alliteration about innocent murmurs in cardiac auscultation. Clin Pediatr (Phila). 2010;49(7):713.

11. Frank JE, Jacobe KM. Evaluation and management of heart murmurs in children. Am Fam Physician. 2011;84(7):793800 .

12. Woo J, Khan O, Caldarelli L, Williams P. Tachycardia in the Neonate. Pediatr Ann. 2015;44(10):e247-e250.

13. Bera D, Ramalingam V, Rathi C, Sharma R, Bachani N, Lokhandwala Y. Arrhythmia in Children and Adolescents and Outcome of Radiofrequency Ablation for Tachyarrhythmias - A Single Center Experience Over 16 Years. Indian Pediatr. 2020;S097475591600200.

14. O'Gara PT, Loscalzo J. Approach to the Patient with a Heart Murmur. In: H Jameson, et. al. (ed). Harrison's Principles of Internal Medicine (20th ed) New York: McGraw-Hill Education. 2018:240-248.

15. Yi MS, Kimball TR, Tsevat J, Mrus JM, Kotagal UR. Evaluation of heart murmurs in children: cost-effectiveness and practical implications. J Pediatr. 2002;141(4):504-511. 